\title{
A hospitalização como fator de seleção em estudo de casos e controles
}

\author{
Carlos Henrique Klein ${ }^{*} \quad$ *Escola Nacional de Saúde \\ Pública - FIOCRUZ - RJ.
}

Neste artigo se demonstra que, se os riscos relativos de hospitalização de expostos em relação aos não-expostos ao fator em investigação nos grupos de comparação hospitalares casos e não-casos da doença-objeto, são diferentes, a estimativa da Razão de Produtos Cruzados $(R P C)$ será distorcida. Essa distorção pode se dar em qualquer direção, para mais ou para menos, quer dizer, tanto a estimativa de risco (RCP) pode ser sub como superestimada, devido ao fator de seleção da hospitalização. Por fim, deriva-se uma expressão algébrica que permite corrigir a estimativa de RPC.

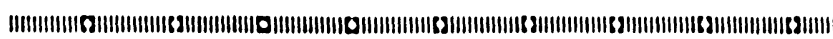

Uma estratégia muito comum nos estudos de casos e controles é a formação dos grupos de comparação com amostras de pessoas hospitalizadas, o que significa que não apenas o grupo de casos é composto por pacientes de um hospital, mas também o de controles ${ }^{2}$.

Vamos considerar a medida chamada de Razão dos Produtos Cruzados (RPC) como indicador de existência e do grau de associação entre o fator e a doença 1 . $\mathrm{O}$ esquema mais simples de um estudo com dois grupos de comparação e um fator dicotômico, isto é de apenas duas categorias (presente e ausente), pode ser representado por:

\begin{tabular}{|c|c|c|c|}
\hline & & $\begin{array}{c}\text { Casos } \\
\text { (C) }\end{array}$ & $\begin{array}{c}\text { Controles } \\
\text { (C) }\end{array}$ \\
\hline \multirow{2}{*}{ Fator } & Presente & $\mathrm{a}$ & $\mathrm{b}$ \\
\hline & Ausente & c & $d$ \\
\hline & Total & $a+c$ & $b+d$ \\
\hline
\end{tabular}

Razão dos Produtos Cruzados RPC $=\frac{\mathrm{a}}{\mathrm{c}} \quad \frac{\mathrm{d}}{\mathrm{b}}$

Recebido para publicação em $13 / 12 / 85$.

Cadernos de Saúde Pública, R.J., 2 (1):13-18,jan/mar,1986. 
As estimativas de Razão dos Produtos Cruzados costumam ser mais baixas nos estudos, cujos controles são pessoas hospitalizadas, uma vez que a distribuição do fator de risco nesses controles se parece mais com a dos casos $^{3}$. Portanto, os controles hospitalizados podem não representar a população de não casos da doençaobjeto e, por isso, a estimativa de risco (RPC) obtida desta maneira não é a mais adequada.

Por outro lado, mesmo que não hajam motivos para suspeitar que os outros doentes, ou pelo menos um grupo determinado destes, difiram dos não-casos da doença-objeto, na população, em relação à distribuição do fator de exposição, ainda assim a hospitalização pode ser um motivo decisivo para a distorção do resultado (RPC). É isto que pretendo neste texto.

A interpretação dos resultados de um estudo de casos e controles com amostras hospitalares deve levar em consideração os riscos de hospitalização, especialmente os de expostos e não-xpostos ao fator em investigação em cada um dos grupos de comparação. Isto quer dizer que, se houver associação entre hospitalização e fator de exposição, os resultados podem não expressar a verdadeira relação entre exposição e doença.

Supondo-se que pode haver uma associação também entre o fator de exposição e a hospitalização, vamos analisar esta relação com o auxílio do Risco Relativo $(R) 1$. O Risco $R e$ lativo, neste caso, é a medida direta de associação entre o fator e a hospitalização. E, com isto, podemos relacionar os riscos relativos de hospitalização para os casos da doençaobjeto e para as outras doenças, que dão origem ao grupo de controles.

Por isto, de modo semelhante ao que é feito para analisar a relação do fator com a doença, vamos ver como se dá a relação entre o fator e a hospitalização na população.

Para os casos da doença-objeto (C), na população:

Fator $\quad$\begin{tabular}{c|c|c|c} 
CASOS & $\begin{array}{c}\text { Hospital } \\
(\mathrm{H})\end{array}$ & $\begin{array}{c}\text { Em casa } \\
(\mathrm{h})\end{array}$ & Todos \\
\hline Presente & ${ }^{a} \mathrm{C}$ & $\mathrm{b}_{C}$ & ${ }^{a} \mathrm{C}+\mathrm{b}_{C}$ \\
\hline Ausente & ${ }^{\mathrm{c}} \mathrm{C}$ & $\mathrm{d}_{\mathrm{C}}$ & ${ }^{{ }^{c}} \mathrm{C}+\mathrm{d}_{C}$
\end{tabular}

O risco relativo de hospitalização dos casos expostos em relação aos não-expostos pode ser dado por:

$$
\mathrm{RC}_{\mathrm{H} / \mathrm{h}}=\frac{\mathrm{a}_{\mathrm{c}}}{\mathrm{c}_{\mathrm{c}}} \quad \frac{\mathrm{c}_{\mathrm{c}}+\mathrm{d}_{\mathrm{c}}}{\mathrm{a}_{\mathrm{c}}+\mathrm{b}_{\mathrm{c}}}
$$


assim que, a razão entre casos expostos e não-expostos que serão realmente hospitalizados pode ser expressa por:

$$
\frac{{ }^{a} \mathrm{C}}{{ }^{c_{C}}}=R C_{H / h} \quad \frac{a_{c}+b_{c}}{c_{c}^{c}+d_{c}}
$$

Do mesmo modo, para as outras doenças hospitalizáveis (D), na população:

\begin{tabular}{|c|c|c|c|c|}
\hline & $\begin{array}{l}\text { OUTRAS } \\
\text { DOENÇAS }\end{array}$ & $\begin{array}{l}\text { Hospital } \\
\text { (H) }\end{array}$ & $\begin{array}{c}\text { Em casa } \\
\text { (h) }\end{array}$ & Todos \\
\hline \multirow{2}{*}{ Fator } & Presente & ${ }^{a} D$ & ${ }^{b} D$ & ${ }^{a} D+b_{D}$ \\
\hline & Ausente & ${ }^{c} D$ & ${ }^{d} D$ & $\mathrm{c}_{\mathrm{D}}+\mathrm{d}_{\mathrm{D}}$ \\
\hline
\end{tabular}

O risco relativo de hospitalização das pessoas com outras doenças, hospitalizáveis, de expostos em relação aos não-expostos pode ser dado por:

$$
\mathrm{RD}_{\mathrm{H} / \mathrm{h}}=\frac{\mathrm{a}_{\mathrm{d}}}{\mathrm{c}_{\mathrm{d}}} \quad \frac{\mathrm{c}_{\mathrm{d}}+\mathrm{d}_{\mathrm{d}}}{\mathrm{a}_{\mathrm{d}}+\mathrm{b}_{\mathrm{d}}}
$$

assim que, a razão entre expostos e não-expostos que serão realmente hospitalizados pode ser expressa por:

$$
\frac{a_{d}}{c_{d}}=R D_{H / h} \frac{a_{d}+b_{d}}{c_{d}+d_{d}}
$$

Portanto, no hospital iremos encontrar realmente indivíduos selecionados dos grupos de casos e controles, de acor-

\begin{tabular}{|c|c|c|c|}
\hline & $\begin{array}{l}\text { No } \\
\text { Hospital }\end{array}$ & $\begin{array}{c}\text { Casos } \\
\text { (C) }\end{array}$ & $\begin{array}{c}\text { Controles } \\
\text { (D) }\end{array}$ \\
\hline \multirow[t]{2}{*}{ Fator } & Presente & ${ }^{a} \mathrm{C}$ & ${ }^{a} D$ \\
\hline & Ausente & ${ }^{c} \mathrm{C}$ & ${ }^{c} D$ \\
\hline
\end{tabular}
do com os riscos de hospitalização. As observações do estudo de casos e controles seriam as seguintes:

Então, a Razão de Produtos Cruzados no Hospital pode ser dada por:

Cadernos de Saúde Pública, R.J., 2 (1):13-18, jan/mar,1986. 


$$
\begin{aligned}
\mathrm{RPCH}_{\mathrm{C} / \mathrm{D}} & =\frac{R C_{H / h}\left(\mathrm{a}_{c}+b_{c}\right)\left(c_{d}+d_{d}\right)}{\left(c_{c}+d_{c}\right) R D_{H / h}\left(a_{d}+b_{d}\right)} \\
& =\frac{R G_{H / h}\left(a_{c}+b_{c}\right)\left(c_{d}+d_{d}\right)}{R D_{H / h}\left(c_{c}+d_{c}\right)\left(a_{d}+b_{d}\right)}
\end{aligned}
$$

Entretanto, se o estudo de casos e controles tivesse sido feito com outras amostras de casos da doença-objeto, e de controles, com outras doenças, retiradas diretamente da população, o quadro de observações seria diferente. Isto porque a cada quantidade observada nas células da tabela do hospital se acrescentariam aqueles indivíduos que não são hospitalizados, mas que, todavia, são também casos $\mathrm{e}$ controles (outros doentes).

A tabela do estudo de casos e controles feito com amostras reoresentativas da população seria a seguinte (sua com-

\begin{tabular}{|c|c|c|c|}
\hline & $\begin{array}{l}\mathrm{Na} \\
\text { População }\end{array}$ & $\begin{array}{l}\text { Casos } \\
\text { (C) }\end{array}$ & $\begin{array}{l}\text { Controles } \\
\text { (D) }\end{array}$ \\
\hline \multirow{2}{*}{ Fator } & Presente & $a_{c}+b_{C}$ & ${ }^{a_{D}}+{ }^{b_{D}}$ \\
\hline & Ausente & ${ }^{c}{ }_{C}+d_{C}$ & ${ }^{c_{D}}+{ }^{d_{D}}$ \\
\hline
\end{tabular}
posição depende de tabelas anteriores):

A Razão de Produtos Cruzados na População, para casos da doença-objeto em relação aos demais doentes será dada por:

$$
\operatorname{RPCP}_{c / d}=\frac{\left(a_{c}+b_{c}\right)\left(c_{d}+d_{d}\right)}{\left(c_{c}+d_{c}\right)\left(a_{d}+b_{d}\right)}
$$

Portanto, podemos redefinir a expressão obtida antes para a Razão de Produtos Cruzados no Hospital, de acordo com esta última, ficando com:

$$
\mathrm{RCPH}_{\mathrm{c} / \mathrm{d}}=\frac{\mathrm{RC}_{\mathrm{H} / \mathrm{h}}}{\mathrm{RD}_{\mathrm{H} / \mathrm{h}}} \quad \mathrm{RPCP}_{\mathrm{c} / \mathrm{d}}
$$

de onde se conclui que a Razão de Produtos Cruzados no Hospital (RPCH) só é igual à Razão de Produtos Cruzados na População (RPCP) quando os riscos relativos de hospitalização, de expostos em relação aos não-expostos, são idênticos nos casos da doença-objeto e nos outros doentes. 
De acordo com estes riscos relativos de hospitalização, o resultado do estudo, no hospital, pode ser distorcido em qualquer direção, para mais ou para menos, em relação ao verdadeiro valor desejado da razão de produtos cruzados. Pois se:

$$
\mathrm{RC}_{\mathrm{H} / \mathrm{h}}>\mathrm{RD}_{\mathrm{H} / \mathrm{h}} \text {, então } \mathrm{RPCH}_{\mathrm{c} / \mathrm{d}}>\mathrm{RPCP}_{\mathrm{c} / \mathrm{d}}
$$

mas, se

$$
\mathrm{RC}_{\mathrm{H} / \mathrm{h}}<\mathrm{RD}_{\mathrm{H} / \mathrm{h}} \text {, então } \mathrm{RPCH}_{\mathrm{c} / \mathrm{d}}<\mathrm{RPCP}_{\mathrm{c} / \mathrm{d}}
$$

Pode-se expressar, também, a Razão dos Produtos Cruzados na população de acordo com os riscos de hospitalização e com a Razão dos Produtos Cruzados no Hospital, da seguinte maneira:

$$
\mathrm{RPCO}_{\mathrm{c} / \mathrm{d}}=\frac{\mathrm{RD}_{\mathrm{H} / \mathrm{h}}}{\mathrm{RC}_{\mathrm{H} / \mathrm{h}}} \quad \mathrm{RPCH}_{\mathrm{c} / \mathrm{d}}
$$

A possibilidade de obtenção de valor corrigido da Razão dos Produtos Cruzados, aquele que se refere à população, depende, como se viu, do conhecimento dos indicadores de risco de hospitalização, de expostos e não-expostos ao fator, nos casos e nos controles. Estes nem sempre são disponiveis e a sua obtenção muitas vezes exige, na prática, novos estudos que podem ser inviáveis. De qualquer modo é sempre útil tentar obter estimativas que avaliem as possibilidades de distorção nos resultados.

$\mathrm{E}$, finalmente, é preciso lembrar, novamente, que a utilização de outros doentes como grupo controle pode, por si só, não ser conveniente. Isto porque estes indivíduos não representam, necessariamente, o total daqueles que não foram acometidos pela doença-objeto em relação a exposição ao fator ${ }^{3}$. É óbvio que a melhor alternativa de formação de grupo controle é aquela que seleciona uma amostra representativa de todos os não-casos da doença-objeto, porém, na prática, isto é quase sempre impossível. Os resultados de estudos de casos e controles hospitalares devem ser vistos, portanto, com muito cuidado e ajuizados com informações de outras fontes.

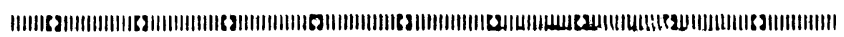

This paper shows that if the relative risks of hospitalization of exposed compared to non-exposed to a putative study factor are different in comparison groups of impatients cases and non-cases of a specific disease the odds-ratio estimate will be biased. This bias can be in 
any direction, either overestimated or underestimated, due to the selection factor of hospitalization. Finally, it is derived an alocbraic expression that can correct the odds-ratio estimate.

\section{REFERENCIAS BIBLIOGRÄFICAS}

1. LILLIENFELD, A.M. Foundations of epidemiology. 2nd. ed., New York, Oxford University Press, 1980.

2. MacMAHON, B \& PUGH, T.F. Epidemiology: Principles and methods. Boston, Little, Brown and Company, 1970.

3. WEST, D.W., SCHUMAN, K.L., LYON, J. L., ROBINSON, L.M. \& ALLRED, R. Differences in risk estimations from a hospital and a population-based case-control study. Int. J. Epidemiology 13(2) : 235-39, 1984. 